\title{
Emulación de una turbina de viento con MPPT en tiempo real*
}

\author{
Julián Peláez-Restrepo** \\ Jorge Herrera-Cuartas ${ }^{* * *}$ \\ Daniel González-Montoya ${ }^{* * * *}$ \\ Sergio Serna-Garcés ${ }^{* * * *}$
}

Recibido: 28/01/2019 • Aceptado: 14/05/2019

https://doi.org/10.22395/rium.v18n35a10

\begin{abstract}
Resumen
En este trabajo se presenta el diseño e implementación de un banco de emulación de turbinas eólicas; el banco propuesto tiene en cuenta la dinámica de la extracción de energía efectiva del viento que se modela matemáticamente. El motor emula el movimiento giratorio debido a la energía del viento, lo cual genera perfiles de velocidad angular según el par medido por un sensor de par en el eje de acople entre el motor DC y el generador síncrono de imanes permanentes. Adicionalmente, se creó un experimento de prueba compuesto por un convertidor elevador controlado al cual se le aplica un algoritmo de extracción de máxima potencia, con el fin de validar el funcionamiento del banco motor DC y el generador síncrono de imanes permanentes al emular la dinámica de un generador eólico equivalente.
\end{abstract}

Palabras clave: emulador de generador eólico; control; MPPT; tiempo-real; electrónica de potencia; fuentes conmutadas; convertidores y accionamientos eléctricos; energía renovable.

Proyecto de investigación para la convocatoria interna del Instituto Tecnológico Metropolitano, P14105 Identificación, modelado y control de una turbina de viento por MPPT.

** Ingeniero electrónico, magíster en Ingeniería Electrónica, doctor en Ingeniería Electrónica. Profesor, Instituto Tecnológico Metropolitano, Medellín, Colombia. Correo electrónico: julianpelaez@itm.edu.co. Orcid: http:// orcid.org/0000-0001-6737-7724

*** Ingeniero electrónico, doctor en Informática Industrial y Técnicas Avanzadas de Producción. Profesor, Departamento de Ingeniería de la Universidad Jorge Tadeo Lozano, Bogotá, Colombia. Correo electrónico: jorgea. herrerac@utadeo.edu.co. Orcid: http://orcid.org/0000-0003-0273-4043

**** Ingeniero de control, magíster en Automatización Industrial, doctor en Ingeniería Automática. Profesor ocasional, Instituto Tecnológico Metropolitano, Medellín, Colombia. Correo electrónico: danielgonzalez@itm.edu.co. Orcid: http://orcid.org/0000-0002-8658-614X

***** Ingeniero electrónico, magíster en Ingeniería de Sistemas, doctor en Ingeniería Automática. Profesor Asistente, Departamento de Electrónica y Telecomunicaciones, Instituto Tecnológico Metropolitano, Medellín, Colombia. Correo electrónico: sergioserna@itm.edu.co. Orcid: http://orcid.org/0000-0002-1498-7323 


\title{
Real-Time Emulator of Wind Turbine with MPPT
}

\begin{abstract}
In this paper, the design and implementation of a wind turbine emulator bank is proposed. The bank takes into account the dynamics of the extraction of effective wind energy. This dynamic is modelled mathematically. The DC motor emulates the rotary movement due to wind energy, generating the angular velocity profiles according to the torque measured by a torque meter on the coupling shaft between the DC motor and the permanent magnet synchronous generator. Additionally, the paper presents a test experiment that consists of a controlled boost converter to which a maximum power extraction algorithm is applied in order to validate the operation of the DC motor-permanent magnet synchronous generator bank.
\end{abstract}

Keywords: wind generator emulator; control; MPPT; real-time; power electronics; switching power supply; converters and electric drives; renewable energy.

\section{Emulação de uma turbina de vento com MPPT em tempo real}

\section{Resumo}

Neste trabalho, apresenta-se o desenho e implementação de um banco de emulação de turbinas eólicas; o banco proposto leva em consideração a dinâmica da extração de energia efetiva do vento que é modelada matematicamente. O motor emula o movimento giratório devido à energia do vento, o que gera perfis de velocidade angular de acordo com o par medido por um senso de par no eixo de encaixe entre o motor DC e o gerador síncrono de imãs permanentes. Adicionalmente, criou-se um experimento de prova composto por um conversor elevador controlado ao que é aplicado um algoritmo de extração de máxima potência, com o objetivo de validar o funcionamento do banco motor DC e do gerador síncrono de imãs permanentes ao emular a dinâmica de um gerador eólico equivalente.

Palavras-chave: emulador de gerador eólico; controle; MPPT; tempo real; electrónica de potência; fontes comutadas; conversores e acionamentos eléctricos; energia renovável. 


\section{INTRODUCCIÓN}

Alrededor del mundo en la última década se ha incrementado de forma considerable la investigación relacionada con energías renovables (extracción, control, distribución, optimización, entre otros) debido principalmente a dos razones: por un lado, el incremento ininterrumpido de consumo de energía de las sociedades modernas; y por otra parte y como consecuencia directa de la anterior, se genera la necesidad mundial de eliminar la huella de carbono para mitigar los efectos del cambio climático [1-2].

La energía eólica es una de las energías renovables que ha ganado fuerza en los últimos años. Para hacerse una idea, al final del 2015 se tenía una capacidad instalada a nivel mundial del orden de los $433 \mathrm{GW}$ [2], esto debido a su gran madurez técnica, bajo costo y no presentar emisión de gases, en comparación con otras fuentes renovables [4-5].

La turbina de viento es el dispositivo mecánico que convierte el flujo del viento en energía de rotación, que finalmente se convierte en energía eléctrica a través de una maquina eléctrica [4]. Las turbinas de viento se encuentran con salidas de potencia en rango de los vatios a los MW; particularmente, se considera una turbina pequeña si es menor a $100 \mathrm{~kW}$. Las turbinas pequeñas se utilizan en aplicaciones de bajo consumo (uso residencial y consumo propio), el problema con estas turbinas es que generalmente no poseen regulación de velocidad, lo cual dificulta el control de la potencia de salida [5]. Dicho control se debe realizar mediante una interfaz electrónica de potencia que se encarga de regular la energía generada por la turbina para garantizar una máxima extracción de potencia [6].

Para desarrollar los convertidores electrónicos usados en el control de estas turbinas es necesario utilizar emuladores de las turbinas de viento (WTE, por sus siglas inglés) para emular su comportamiento en pruebas de laboratorios [7]. Con los WTE se pueden probar los diseños tanto de los convertidores como de los algoritmos que se encargan de realizar un seguimiento de la condición optima de la turbina ante distintas condiciones ambientales, también llamado búsqueda del máximo punto de potencia (MPPT, por sus siglas en inglés) $[8,10]$.

Las pruebas ante distintas condiciones ambientales son necesarias ya que la energía del viento es impredecible y por lo tanto difícil de manipular en el laboratorio, debido a que no existe un perfil de viento estándar para un día de operación; es decir que el viento posee un comportamiento aleatorio que dificulta el conocimiento a priori de la condición de operación óptima de la turbina. Sin embargo, se pueden conocer los rangos típicos de los perfiles de viento de un lugar geográfico. A modo de ejemplo, 
Colombia cuenta con el mejor potencial eólico en la península de la Guajira por sus velocidades de viento que varían entre 7,4 y $16,6 \mathrm{~m} / \mathrm{s}$ [11-12].

Simular las condiciones promedio que se podrían dar en un lugar determinado implica adoptar una de dos opciones para el diseño del sistema de procesamiento de energía en la turbina. La primera opción es tener un túnel de viento perfectamente acoplado al tamaño de la turbina que se desea someter a prueba. Esta condición se dificulta ya que la instrumentación de este tipo de soluciones es compleja para garantizar la velocidad del viento requerida en la prueba específica. La segunda opción depende de implementar un banco de emulación con un acople mecánico entre un motor-generador. El generador es tipo síncrono de imanes permanentes (PMSG) trifásico, el cual se utiliza comúnmente en aplicaciones eólicas de pequeña escala (en adelante, se le menciona sencillamente como 'generador'), el motor es un motor DC (en adelante, se le alude sencillamente como 'motor'). El emulador tiene en cuenta la curva típica del coeficiente de potencia $C_{P}(\lambda, \beta)$ de una turbina específica que depende principalmente del ángulo de ataque del viento y el radio. Este emulador permite probar en diferentes velocidades de viento la generación de energía de diversos tamaños de turbinas, sin la necesidad de instrumentación compleja que garantice una correcta velocidad del viento.

Por tanto, este artículo presenta el diseño e implementación de un sistema de generación de energía eólica (WECS) basado en el emulador de turbinas de viento [8]. El WECS en mención se compone de dos máquinas eléctricas acopladas mecánicamente, una actúa como un motor que emula el movimiento rotacional debido a las dinámicas del viento en la turbina y la otra actúa como el generador [10].

\section{DINÁMICAS DEL VIENTO EN TURBINAS EÓLICAS}

Entender las dinámicas del viento en las turbinas eólicas es de gran importancia, ya que estas tienen una eficiencia superior dada por las restricciones que impone el límite de Betz [11]; es decir que ninguna turbina eólica de sección transversal de tres palas tiene una eficiencia mayor a la máxima eficiencia posible de acuerdo con la curva del coeficiente de potencia $(0,593)$, esto es, cuánta de la potencia efectiva del viento se logra transferir a potencia mecánica en el rotor de la turbina; esta restricción se encuentra explicada físicamente en [11]. Después de múltiples pruebas con rotores conectados a turbinas de tres palas y de realizar un barrido de velocidades de viento, se obtienen las gráficas del coeficiente de potencia versus velocidad de punta de pala a diferentes ángulos de $\hat{\boldsymbol{a}}$, como se muestra en la figura 1 . 


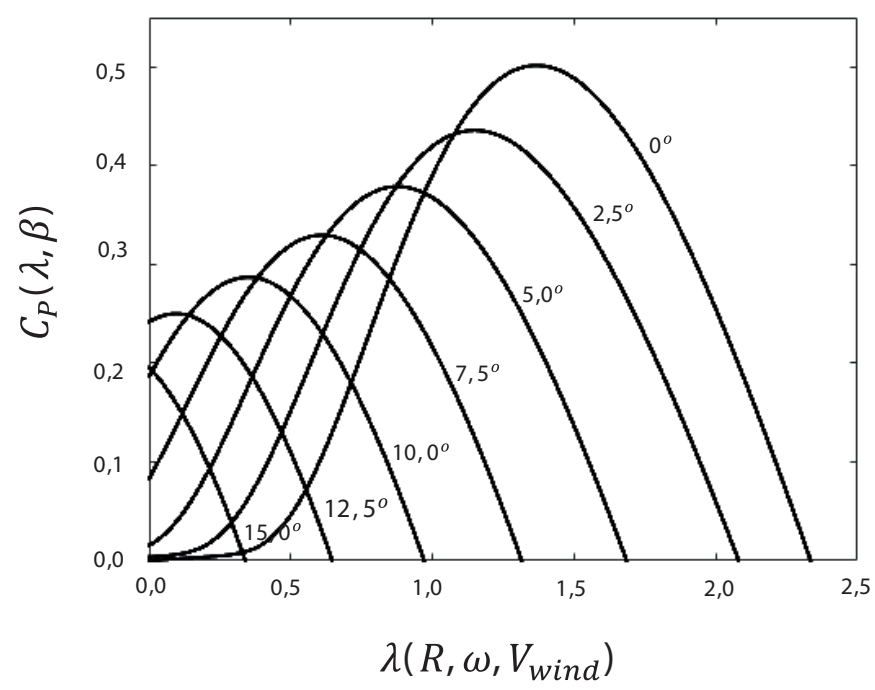

Figura 1. Coeficiente de potencia característico

Fuente: elaboración propia

Igualmente, el modelo general del rotor de la turbina (ecuación 1) se tomó de [7], en el cual se presenta la ecuación del coeficiente de potencia (ecuación 2), siendo esta una relación entre la potencia del viento y la potencia mecánica que realmente se transfiere al rotor de la turbina. La ecuación (2) aplica para todas las turbinas de tres palas en las que los parámetros $\boldsymbol{C}_{1}, \boldsymbol{C}_{2}, \boldsymbol{C}_{1}, \boldsymbol{C}_{3}, \boldsymbol{C}_{4}, \boldsymbol{C}_{5} \boldsymbol{y} \boldsymbol{C}_{6}$ se hallaron por medio de un algoritmo de regresión. Asimismo, en la ecuación (2) $\beta$ es el ángulo de ataque de la sección transversal de las palas con respecto a la dirección del viento; en turbinas de pequeña escala este valor es fijo y se suele aproximar a cero ya que, según la figura 1 , este es el ángulo en el que mayor eficiencia alcanza la turbina. $\lambda_{i}$ es una relación representada en la ecuación 3 y $\lambda$ es la velocidad de punta de pala de la turbina que depende proporcionalmente del radio de las palas en $\boldsymbol{m}$. $\omega$ es la velocidad angular del rotor de la turbina con unidades en $\mathrm{rad} / \mathrm{s}$, e inversamente proporcional a la velocidad del viento $\boldsymbol{V}_{\text {wind }}$ en $\mathrm{m} / \mathrm{s}$ como se ve en la ecuación 3:

$$
\begin{gathered}
P_{W G}=\frac{1}{2} \rho_{\text {air }}\left(\pi R^{2}\right) V_{\text {wind }}^{3} C_{p}(\lambda, \beta)=\omega_{r} \tau_{r} \\
C_{p}(\lambda, \beta)=C_{1}\left(\frac{C_{2}}{\lambda_{i}}-C_{3} \beta-C_{4}\right) e^{\left(-\frac{C_{5}}{\lambda_{i}}\right)}+C_{6} \lambda_{i}
\end{gathered}
$$


Donde:

$$
\lambda_{i}=\left[\frac{1}{\lambda+0.089 \beta}-\frac{0.035}{\beta^{3}+1}\right]^{-1}
$$

Para el cálculo de la velocidad de punta de pala $\lambda$ se hace uso de la expresión dada en [12] como se presenta en la ecuación 4, donde $\omega_{r}$ es la velocidad angular del rotor en $(\mathrm{rad} / \mathrm{s})$.

$$
\lambda=\left(\frac{R \omega_{r}}{V_{\text {wind }}}\right)
$$

Para la turbina Air Breeze se halló una curva característica que se puede observar en la figura 1 al hacer un barrido de sus puntos de operación. Posteriormente se aplicói un algoritmo de regresión en Matlab para hallar los valores de los parámetros específicos de la curva que son $\boldsymbol{C}_{1}=4,152 ; \boldsymbol{C}_{2}=4 ; \boldsymbol{C}_{3}=0,06959 ; \boldsymbol{C}_{4}=1,616 ; \boldsymbol{C}_{5}=3,34$; $\boldsymbol{C}_{6}=0,01886$. Teniendo en cuenta que $\beta=0$, ya que es una turbina de baja potencia con rotor fijo en la cual no varía su ángulo; el modelo incluye $\beta$ porque este se aplica a cualquier turbina transversal de tres palas, sin importar su tamaño.

El viento tiene una potencia que viene dada por la ecuación (5) que depende de la velocidad del viento $\boldsymbol{V}_{\text {wind }}$ en $(\boldsymbol{m} / \boldsymbol{s})$, de la densidad del aire $\rho_{\text {air }}$ que es $1,225\left(\boldsymbol{k g} / \boldsymbol{m}^{3}\right)$ y del área transversal $\boldsymbol{A}$ en la cual se va a medir la potencia del viento; que en el caso de una turbina es el área transversal del rotor debido a las palas, es decir, el área de un circulo $\boldsymbol{A}=\pi \boldsymbol{r}^{2}\left(\boldsymbol{m}^{2}\right)$.

$$
P_{\text {wind }}=\frac{1}{2} \rho_{\text {air }} A V_{\text {wind }}^{3}
$$

A partir de la ecuación 1 y la ecuación 5 se calcula cuánta de la potencia del viento dada por la ecuación 5 se convierte en potencia mecánica efectiva en el rotor de la turbina, la cual -como se ha demostrado en muchos trabajos- se obtiene como producto entre la potencia del viento $\boldsymbol{P}_{\text {wind }}$ y el coeficiente de potencia $\boldsymbol{C}_{\boldsymbol{p}}(\lambda, \beta)$; la máxima potencia que se logra convertir en potencia mecánica-ecuación 5-será, en el mejor de los casos, $0,593 \boldsymbol{P}_{\text {wind }}$; sin embargo, también se sabe que la potencia mecánica de un rotor es el producto del par $\tau_{\boldsymbol{r}}(\boldsymbol{n m})$ por la velocidad angular del rotor $\omega_{\boldsymbol{r}}(\boldsymbol{r a d} / \boldsymbol{s})$ . Así que el algoritmo MPPT modifica el punto de operación de la turbina para que se ubique en el máximo punto de la curva de la figura 1.

\section{EMULACIÓN DE TURBINA DE VIENTO}

La emulación de una turbina eólica debe estar configurada, tal como se presenta en la figura 2. Dicho esquema presenta un acople motor-generador para la emulación 
de una turbina de viento que considere las características propias del sistema. Este sistema requiere del cálculo de la velocidad angular de referencia del motor dado por la ecuación 6.

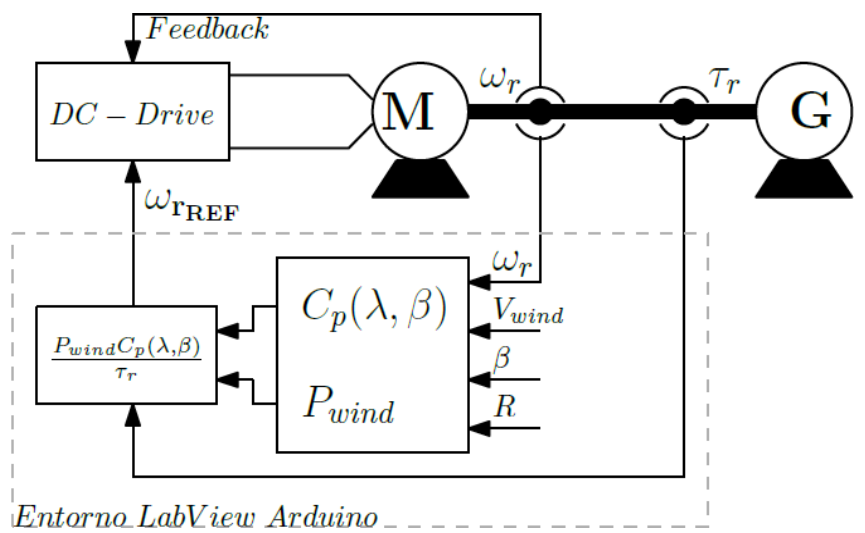

Figura 2. Interfaz del emulador de la turbina eólica

$$
\begin{aligned}
& \text { Fuente: elaboración propia } \\
& \omega_{\text {ref }}=\frac{\left(P_{\text {wind }} C_{P}(\lambda, \beta)\right)}{\tau_{r}}
\end{aligned}
$$

Para ello, el sistema requiere de la adquisición de la medida del par $\tau_{r}$ ( $\mathrm{nm}$ newton metro), del cálculo de potencia generada por el viento $P_{\text {wind }}(W$ vatios) y del coeficiente de potencia $C_{P}(\lambda, \beta)$ (adimensional), como se indica en [13]. Asimismo, el cálculo de la velocidad de referencia $\omega_{\text {ref }}(\mathrm{rad} / \mathrm{s})$ se debe aplicar a la tarjeta de control de velocidad del motor DC para garantizar una correcta emulación de las condiciones de viento requeridas.

Es de notar que el procesamiento de la información medida del acople motorgenerador se debe procesar en tiempo real por medio de un sistema de adquisición que le permita hacer las variaciones necesarias para emular condiciones reales. Esta interfaz se puede implementar con dispositivos de procesamiento de señales típicos (tarjetas de adquisición, DSP, Arduino, etc.). Como se presenta en secciones posteriores, el sistema implementado para la plataforma de emulación consiste en un Arduino UNO como tarjeta de adquisición, que a su vez envía los datos a LabVIEW para su procesamiento e interacción.

En la figura 3 se puede apreciar el procedimiento para emular el sistema eólico completo. Su principal entrada es la velocidad especifica de viento que se quiere emular y está dada por el usuario, también se da por las constantes del sistema como los 
parámetros de $C_{p}(\lambda, \beta)$, el radio del rotor $R$ y la densidad del aire $\rho_{\text {aire }}$. Hay variables que también se ingresan pero que no son dadas por el usuario sino que se adquieren por medio de sensores, como la medida del par y la velocidad angular y, finalmente, la salida es la velocidad de referencia que se aplica a la tarjeta que controla la velocidad del motor DC $\omega_{\text {ref }}$.

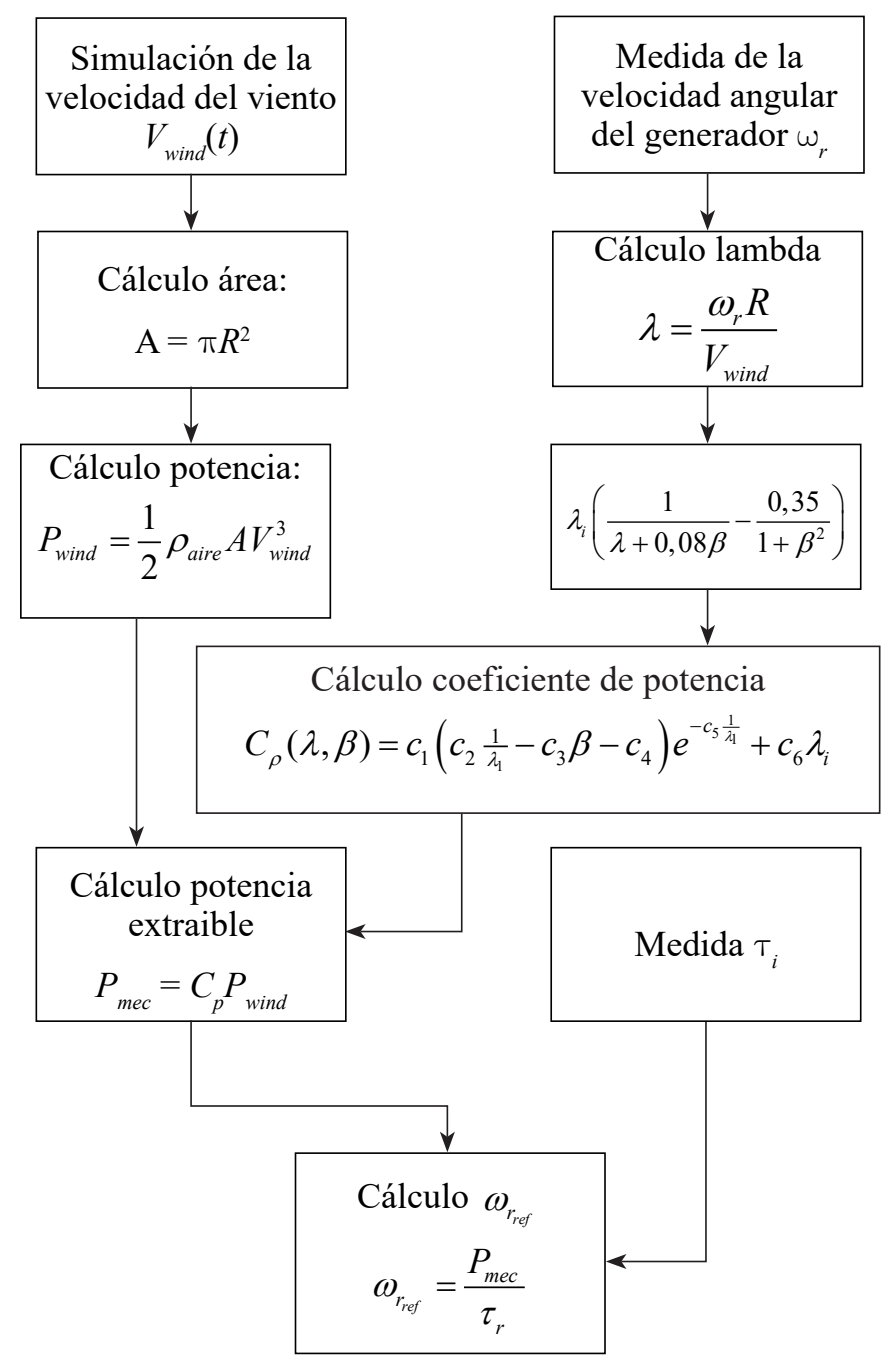

Figura 3. Procedimiento para emular la turbina de viento

Fuente: elaboración propia 


\section{Modelamiento matemático del convertidor conmutado}

Debido a que la turbina de viento genera voltajes inferiores a los demandados por la carga, el convertidor propicio para la aplicación es un convertidor DC-DC elevador, el cual se acopla después de un puente rectificador trifásico con su respectivo condensador de filtrado $C_{i n}$ [14]. Este convertidor tiene como función principal acondicionar la potencia de la turbina y en este caso, entregársela a una carga resistiva, dependiendo de unos niveles de tensión requeridos en el capacitor de salida $C_{o}$; típicamente el control que se diseña para este tipo de aplicaciones es el Control Proporcional Integral (PI) [15].

El convertidor DC-DC elevador [16] de la figura 4, cuyo modelo matemático es dado por el modelo promediado, tiene tres elementos activos que son: la inductancia, el capacitor de entrada y el capacitor de salida, los cuales están asociados a los tres estados del convertidor: la corriente de la bobina $i_{L}(t)$; el voltaje del capacitor de entrada $v_{C_{i n}}(t)$; y el voltaje del capacitor de salida $v_{C_{0}}(t)$.

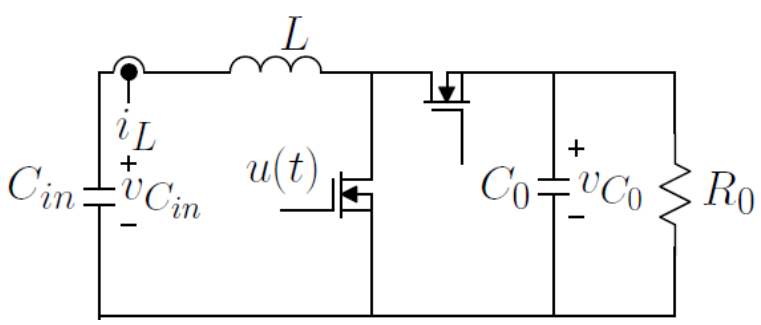

Figura 4. Convertidor dc-dc elevador

Fuente: elaboración propia y modificación a partir de [19]

A partir del modelado de convertidores con propósitos de control presentados en [17] se puede obtener una representación matemática en espacio de estados, como se muestra en la ecuación 7.

$$
\dot{x}(t)=\left[\begin{array}{ccc}
0 & -\frac{1}{C_{i n}} & 0 \\
\frac{1}{L} & 0 & -\frac{(1-d)}{L} \\
0 & \frac{(1-d)}{C_{0}} & -\frac{1}{R_{0} C_{0}}
\end{array}\right] x(t)+\left[\begin{array}{c}
-\frac{i_{i n}}{C_{i n}} \\
0 \\
0
\end{array}\right]
$$

Para este caso, el espacio de estados se presenta en función de regular la extracción de potencia del aerogenerador, es decir que se desea controlar la corriente de entrada al convertidor asociada a la corriente de la bobina $i_{L}(t)$. 
Asimismo, los estados del sistema $x(t)$ están conformados por $v_{C_{i n}}(t), i_{L}(t) \mathrm{y} v_{C_{o}}(t)$.

Teniendo en cuenta la no linealidad del sistema, se requiere una aproximación lineal al modelo evaluado en un punto de equilibrio para garantizar el diseño correcto de un controlador $P I$, como se propone en [18]. Los puntos de equilibrio del sistema se pueden predecir cuando el convertidor se ha estabilizado, es decir cuando $\dot{x}(t)=0$ , así $V_{C_{i n}}^{*}=(1-D)^{2} R_{0} i_{i n}, I_{L}^{*}=i_{i n}, V_{C_{0}}^{*}=(1-D) R_{0} i_{i n}$. Estos valores se reemplazan en el modelo lineal evaluado en los puntos de equilibrio.

A partir del modelo presentado en la ecuación 8 , se procede a diseñar un controlador PI que cumpla con los criterios deseados para la regulación de la corriente del convertidor a partir de una referencia dada por un algoritmo MPPT.

$$
\dot{x}(t)=\left[\begin{array}{ccc}
0 & -\frac{1}{C_{i n}} & 0 \\
\frac{1}{L} & 0 & -\frac{(1-D)}{L} \\
0 & \frac{(1-D)}{C_{0}} & -\frac{1}{R_{0} C_{0}}
\end{array}\right] x(t)+\left[\begin{array}{c}
0 \\
\frac{V_{C_{0}}^{*}}{L} \\
-\frac{I_{L}^{*}}{C_{0}}
\end{array}\right] d(t)
$$

\section{Diseño de algoritmo MPPT}

Una de las técnicas más utilizadas en la literatura para la maximización de la extracción de potencia en fuentes de energía renovables es el algoritmo de perturbar y observar (P\&O) [22-23]. Este algoritmo aplica cambios en la referencia de corriente que se le demanda a la turbina; en caso de que la potencia demandada aumente, el algoritmo sigue perturbando en la misma dirección hasta que finalmente la potencia no aumenta más y se queda atrapado en un ciclo límite alrededor del punto de máxima potencia. Sin embargo, estas variaciones de las referencias se deben hacer de forma tal que el sistema alcance a estabilizarse. Teniendo en cuenta que esta demanda de potencia afecta las dinámicas, tanto de la turbina como del convertidor, en una turbina de viento el cambio hecho por el MPPT P\&O debe tener una dinámica lenta, debido a que las variaciones tienen componentes con dinámicas debidas a fenómenos mecánicos y eléctricos. El algoritmo MPPT P\&O implementado en este trabajo se realizó en un DSP TMS320F28335 y tiene la lógica del diagrama de flujo de la figura 5. 


\section{Implementación de plataforma de emulación de turbina de viento con algoritmo MPPT}

Se cuenta con un banco de motor-generador, el motor DC está controlado por medio de un controlador de velocidad del fabricante Baldor, este mide la realimentación por medio de un generador que hace las veces de sensor de velocidad y entrega un voltaje DC proporcional a la velocidad del rotor. El generador está acoplado al motor en la parte trasera, además, el banco cuenta con un medidor de velocidad fabricado con un disco de ranuras y un opto-acoplador cuya función es medir la velocidad del rotor aislado de la tarjeta de potencia y un medidor de par marca Futek TSR600 de máximo 5 N.m., como se muestra en la figura 6 .

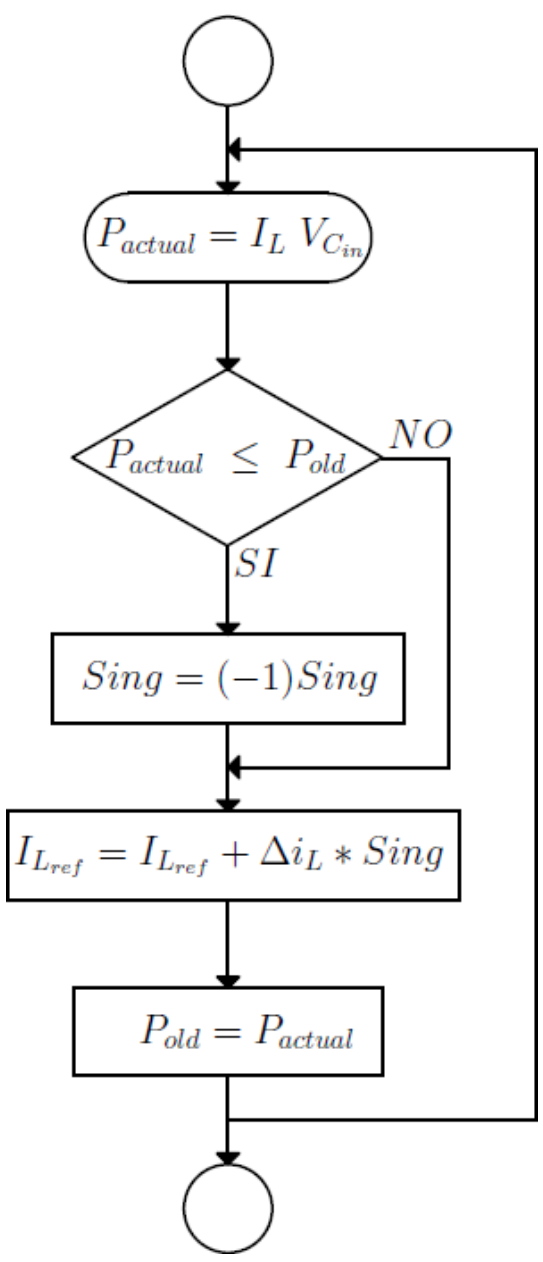

Figura 5. Algoritmo MPPT P\&O clásico

Fuente: elaboración propia 


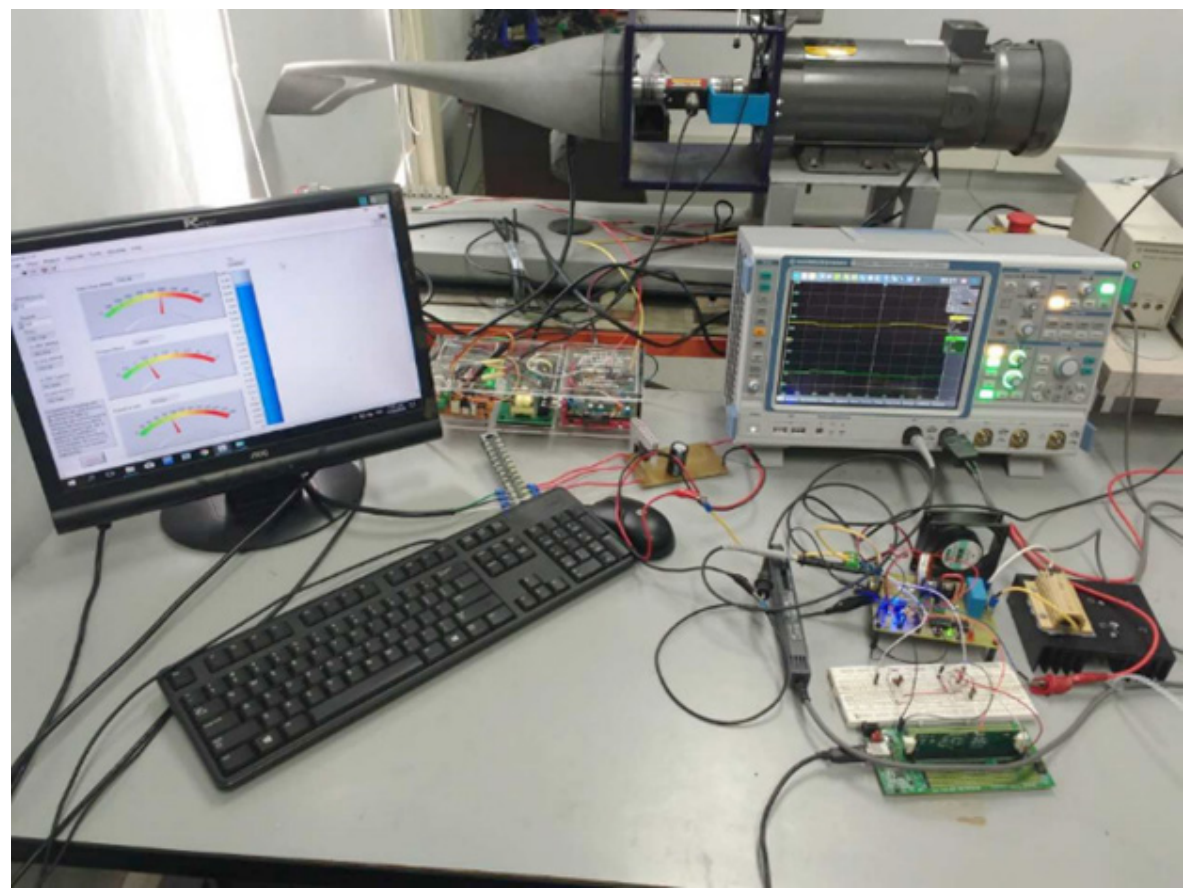

Figura 6. Foto del emulador eólico

Fuente: elaboración propia

A nivel experimental se cuenta con una turbina de viento marca Air Breeze de $200 \mathrm{~W}$, la cual permite emular cualquier tipo de turbina eólica que tenga una velocidad de rotor menor a $1.000 \mathrm{RPM}$ y una potencia menor a $200 \mathrm{~W}$. Por lo tanto, la dinámica de extracción de potencia del viento se simula desarrollando un control de la potencia mecánica entregada por el motor DC al generador PMSG.

Como se mencionó anteriormente, se requiere de una plataforma que procese la información de la plataforma motor-generador que emule las características necesarias para garantizar un correcto funcionamiento del sistema. Dicha plataforma se diseña como se presenta en la figura 7, en la cual, por medio de una interfaz programada con LabVIEW y Arduino, es posible simular el comportamiento de la turbina a un perfil de viento determinado según la curva del coeficiente de potencia, esto se logra al integrar un sensor de velocidad angular, un sensor de par y el driver del motor DC con la interfaz gráfica, la cual entrega al driver del motor una velocidad angular de referencia para un punto de operación dado, a la cual se le debe entregar la velocidad del viento que se desea simular y la potencia nominal de la turbina a emular (ver figura 2). 


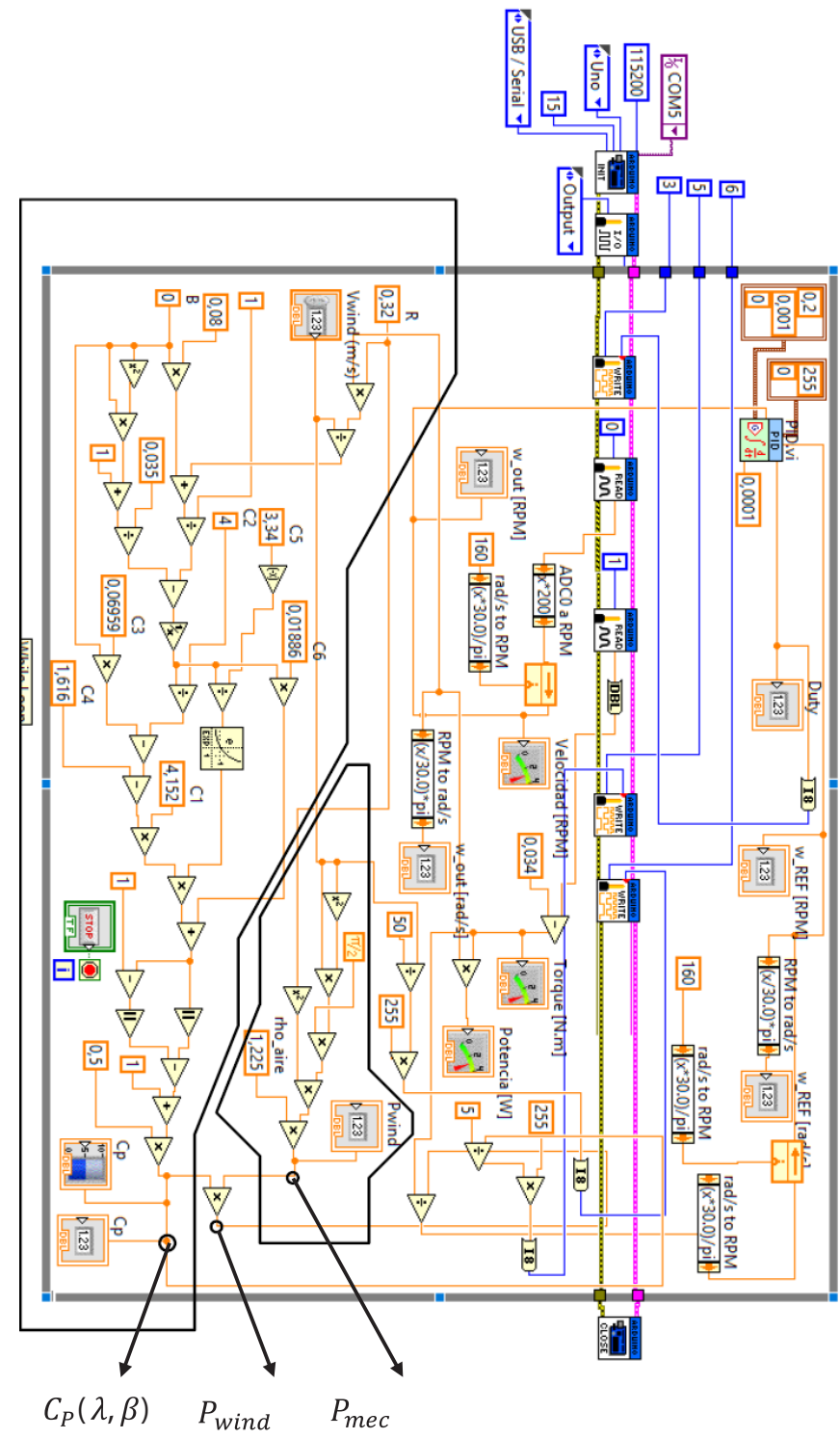

Figura 7. Programa en LabVIEW del emulador eólico

Fuente: elaboración propia

Los valores de velocidad angular y par se adquirieron con un microcontrolador Arduino como tarjeta de adquisición de datos, en el cual la lectura se realiza por medio de cada uno de los puertos ADC; asimismo, se reproducen las señales análogas con puertos PWM filtrados, necesarias para monitorear la velocidad angular de referencia $\omega_{\text {ref }}$ y el coeficiente de potencia $C_{P}(\lambda, \beta)$. 
También se configuró una interfaz gráfica, como se presenta en la figura 8, en la cual se muestra en tiempo real y en pantalla los valores de las variables que se están midiendo. Esta interfaz permite como entrada la velocidad del viento y se encarga de evaluar la velocidad real generada por la turbina, el punto de operación $C_{p}$ alcanzado por la turbina, y muestra los valores de velocidad angular tanto en $R P M$ como en $\frac{\mathrm{rad}}{\mathrm{s}}$.

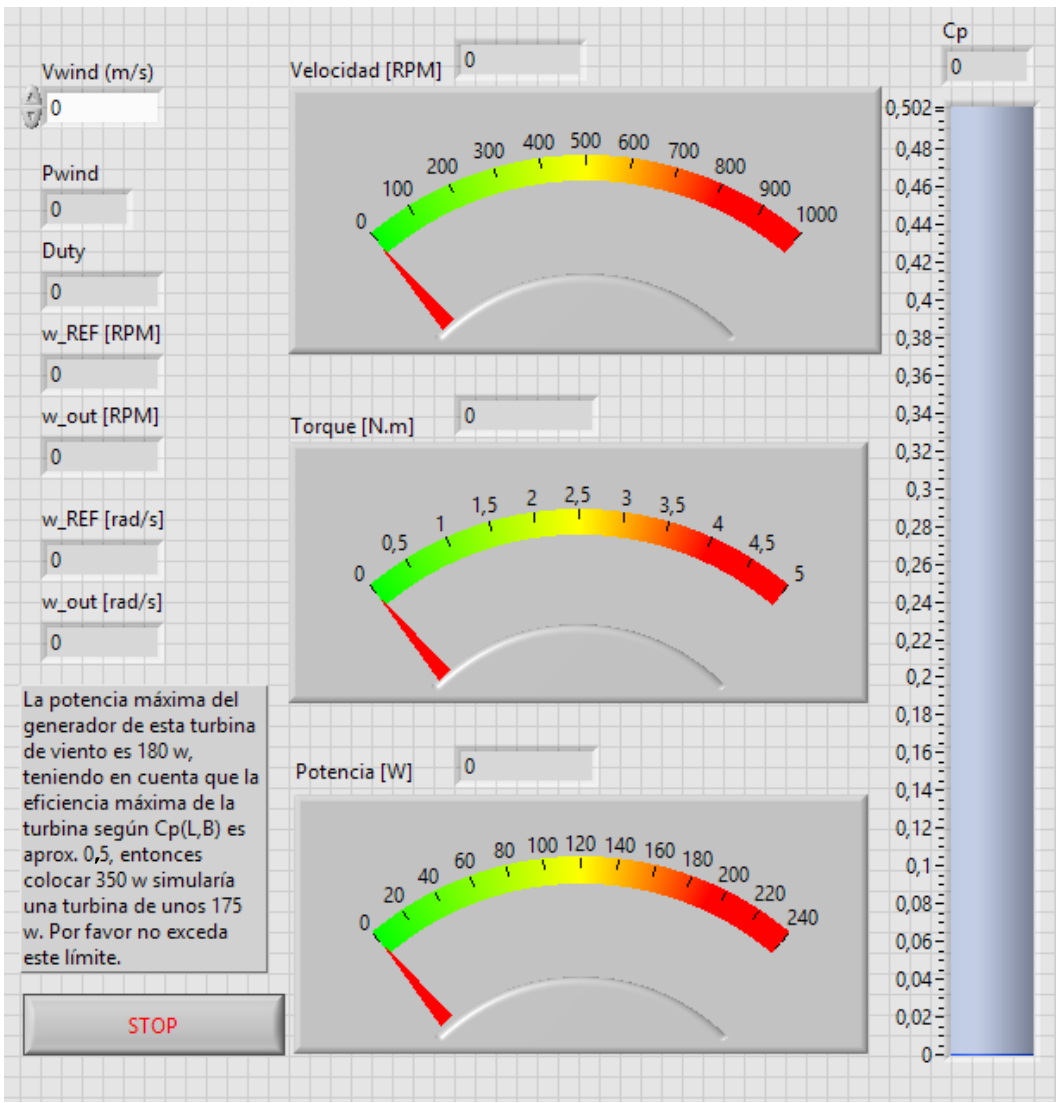

Figura 8. Interfaz en LabVIEW del emulador eólico

Fuente: elaboración propia

Además de mostrar gráficamente las mediciones instantáneas de la velocidad angular en $R P M$ y rad/seg, del par (nm), también se muestra la potencia del viento extraída y el valor del coeficiente de potencia $C_{P}(\lambda, \beta)$.

Finalmente, la figura 9 presenta la plataforma completa de emulación considerando la interfaz de electrónica de potencia y el algoritmo MPPT. 


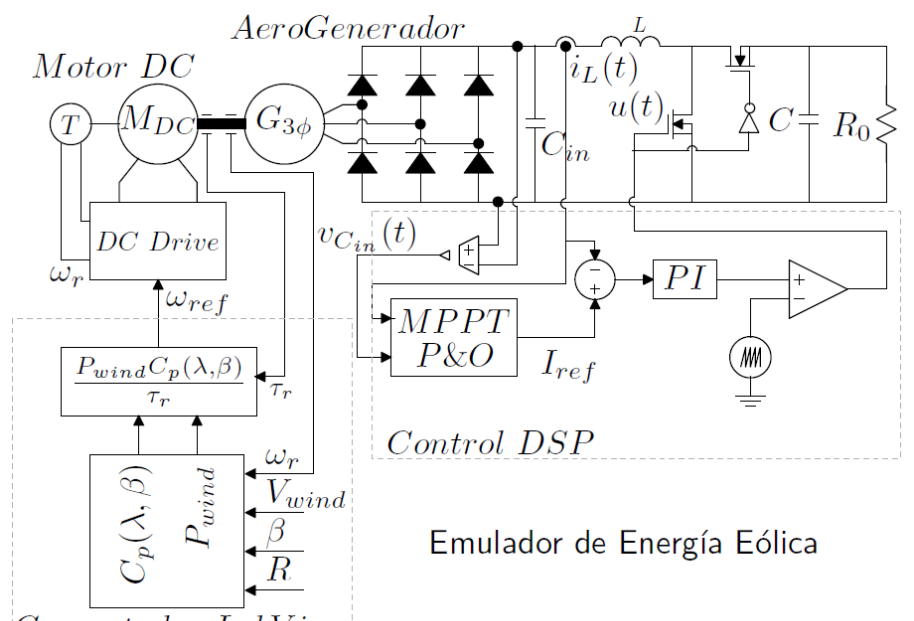

Computador LabView

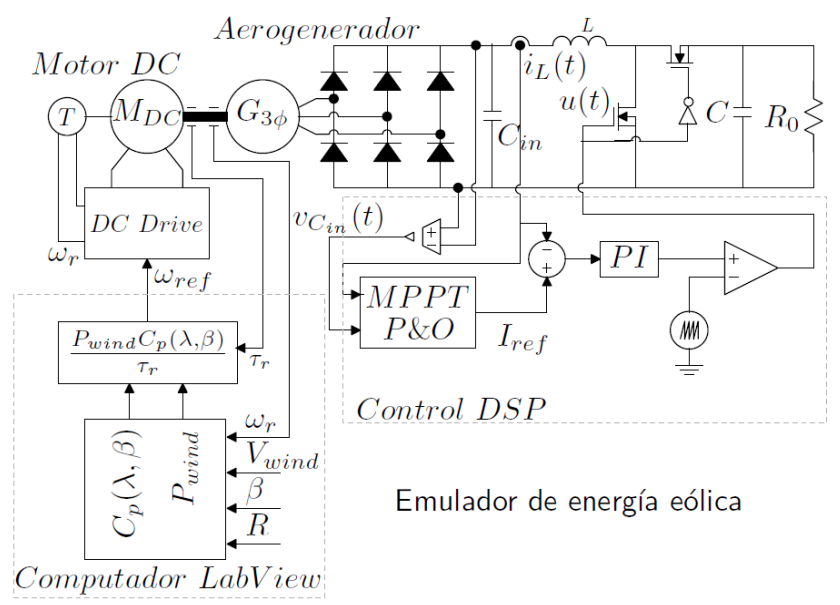

Figura 9. Emulador sistema aerogenerador completo

Fuente: elaboración propia

Conectado a las terminales del generador se tiene un puente rectificador trifásico el cual posee un condensador de filtrado de la señal rectificada y a continuación, un convertidor tipo Boost el cual se controla con un compensador PI para regular corriente. Dicho controlador se encuentra diseñado para seguir las referencias de corriente que entrega el algoritmo MPPT en la búsqueda de un máximo punto de potencia; tanto el control como el algoritmo MPPT se implementan en la DSP TMS320F28335. 


\section{RESULTADOS EXPERIMENTALES}

Se configuró la plataforma experimental para emular unas condiciones particulares en las que los coeficientes para el cálculo del coeficiente de potencia son: $C_{1}=4,152$, $C_{2}=4, C_{3}=0,06959, C_{4}=1,616, C_{5}=3,34, C_{6}=0,01886$. Estos parámetros permiten emular la turbina con distintas velocidades. En este primer intento se simuló una velocidad del viento de $V_{\text {wind }}=12,5 \frac{\mathrm{m}}{\mathrm{s}}$ que a su vez expone un coeficiente de potencia como el de la figura 1.

Sin embargo, para efectos prácticos la curva se tuvo en cuenta solamente cuando su ángulo $\beta=0^{\circ}$ debido a que es una turbina pequeña de palas fijas en la que el coeficiente de potencia óptimo se encuentra alrededor de 0,5 que es el máximo valor del coeficiente de potencia que se logra en el montaje real, es hacia esto que debe tender el MPPT.

Para que el coeficiente de potencia alcance a ser el óptimo, es necesario que haya un algoritmo de búsqueda del punto de máxima potencia. Estos algoritmos deben ser adaptados para el tipo de fuente de energía utilizado, en energía eólica es bastante complicado ajustarlos debido a la naturaleza mecánica del sistema que introduce retardos y vibraciones debido a los componentes mecánicos, lo cual altera la búsqueda estable del punto de máxima potencia; además, las curvas no cambian solo con el ángulo $\beta$ sino también pueden cambiar por $\lambda$, el cual depende de parámetros como la velocidad del viento $V_{\text {wind }}$, la velocidad angular $\omega_{r}$ y la longitud de las palas $r$. El banco utilizado permite diseñar bajo condiciones ideales algoritmos MPPT y realizar ajustes a los algoritmos MPPT que se realicen con otras técnicas como por ejemplo, al aplicar optimización, IA, etc.

A partir del procedimiento presentado en [18], se diseñó un controlador PI para el lazo de corriente de un convertidor elevador, el modelo en el dominio del tiempo discreto basado en la transformada $z$ se obtuvo al despejar desde el modelo lineal de la ecuación 8 las funciones de transferencia de cada una de las variables de estado, que están dadas por $X(z)=\operatorname{inv}(z \boldsymbol{I}-\boldsymbol{A}) \cdot \boldsymbol{B} D(z)$ y remplazar los valores de los parámetros del modelo por valores numéricos, los cuales están dados por: $I_{L}^{*}=6 \mathrm{~A}, V_{C_{0}}^{*}=30 \mathrm{~V}$, $V_{C_{i n}}^{*}=30 \mathrm{~V}, C_{i n}=4700 \mu \mathrm{F}, C_{0}=22 \mu \mathrm{F}, L=330 \mu \mathrm{H}, R_{0}=10 \Omega$, de allí se obtiene la ecuación 9.

Las funciones de transferencia que describen las dinámicas de la corriente de la bobina con respecto a control $\frac{I_{L}(z)}{d(z)}$, muestreadas a $100 \mathrm{kHz}$ se presenta en 9. 


$$
\frac{I_{L}(z)}{d(z)}=\frac{-2,563(z-1,001)(z-1,087)}{(z-0,9992)\left(z^{2}-1,953 z+0,9564\right)}
$$

La idea de implementar un algoritmo de búsqueda del máximo punto de potencia, tipo $\mathrm{P} \& \mathrm{O}$, requiere que la variable entregada por este sirva como referencia de un controlador de un convertidor conmutado -en este caso es un control de corriente-, el control es un controlador PI en tiempo discreto, ya que se implementa en un procesador digital de señales, junto con el algoritmo MPPT. Basado en la función de transferencia dada anteriormente con ayuda del Toolbox de Matlab SiSotool, se sintonizó el controlador PI descrito en la ecuación (10).

$$
=-0,0003 \frac{(z-0,99)}{(z-1)}
$$

Asimismo, el MPPT P\&O implementado se codificó con los siguientes parámetros: $\Delta I_{\text {ref }}=0.5 \mathrm{~A}$, y un $\Delta T_{\text {step }}=8 \mathrm{~s}$ debido a que el tiempo de estabilizacion debe ser suficiente para que el sistema mecánico y eléctrico alcancen el estado estable.

En la figura 10 se observan los resultados experimentales de la plataforma propuesta. La grafica presenta las cuatro variables significativas que demuestran el buen comportamiento del sistema: voltaje de entrada del convertidor (amarillo), corriente de entrada del convertidor (rosa), en el color azul claro o magenta se puede ver la velocidad del viento simulada (magenta) y el coeficiente de potencia en el cual está operando la turbina (azul).

Los resultados experimentales de la figura 10 presentan el desempeño del algoritmo MPPT ante cambios en la velocidad del viento, donde se conserva la extracción del punto de máxima potencia ante dichas variaciones. Asimismo, el coeficiente de potencia se conserva en el valor esperado de 0,5 ; aunque cambie la velocidad del viento, se observó que el algoritmo mueve la corriente de referencia, llevando así el coeficiente $C_{p}$ a su máximo posible, como se ilustra en la figura 10.

La figura 11 presenta el comportamiento detallado del sistema cuando el viento se estabiliza, allí se observa que el MPPT tiende a estar más estable dentro los tres puntos de operación en los que debería oscilar cuando encuentra un máximo. Las regiones en las cuales el algoritmo tiene un comportamiento de más o menos puntos se deben al ruido de medida de los sensores de corriente y voltaje implementados en el sistema. 

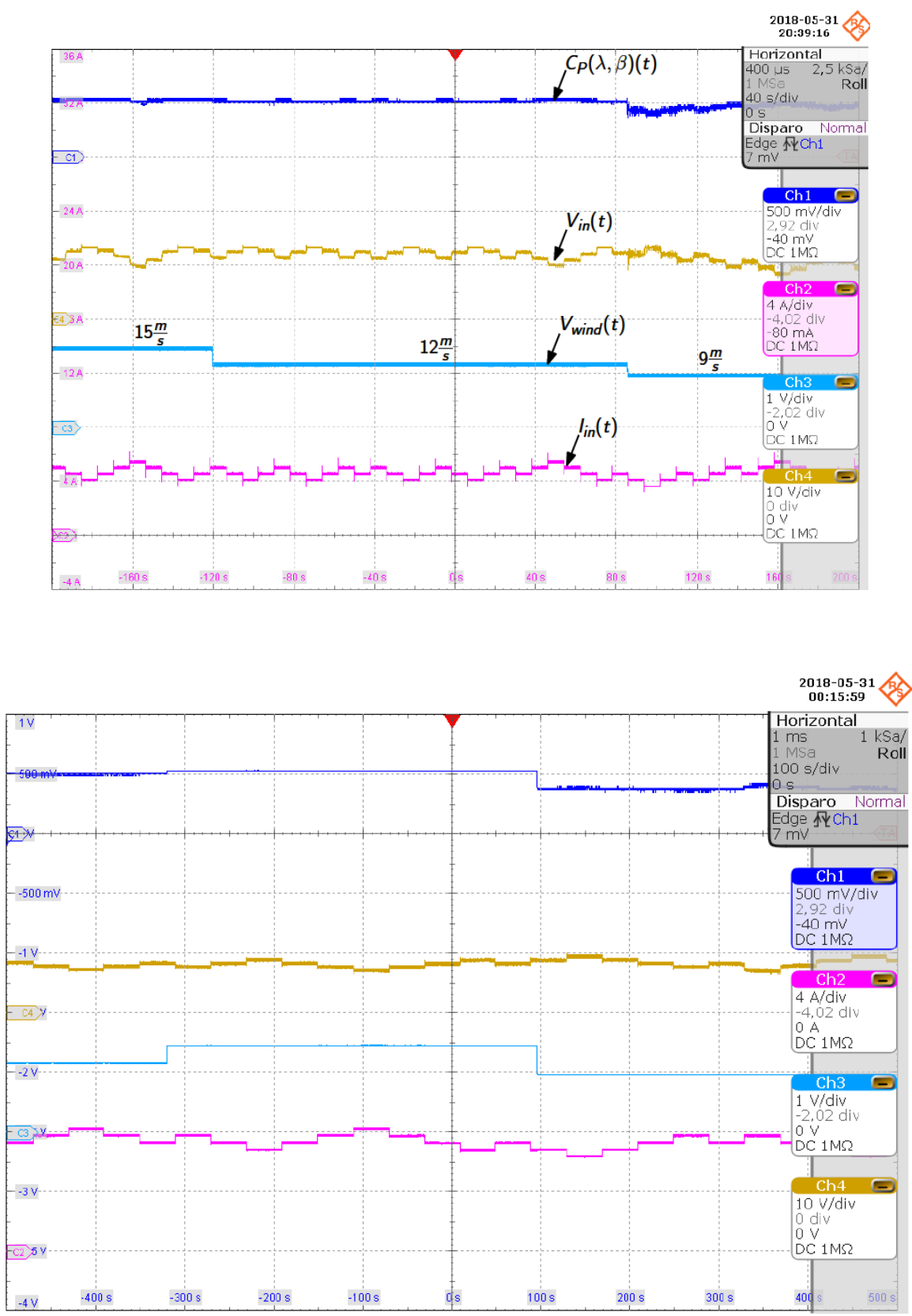

Figura 10. Prueba experimental de un MPPT con el emulador eólico

Fuente: elaboración propia 


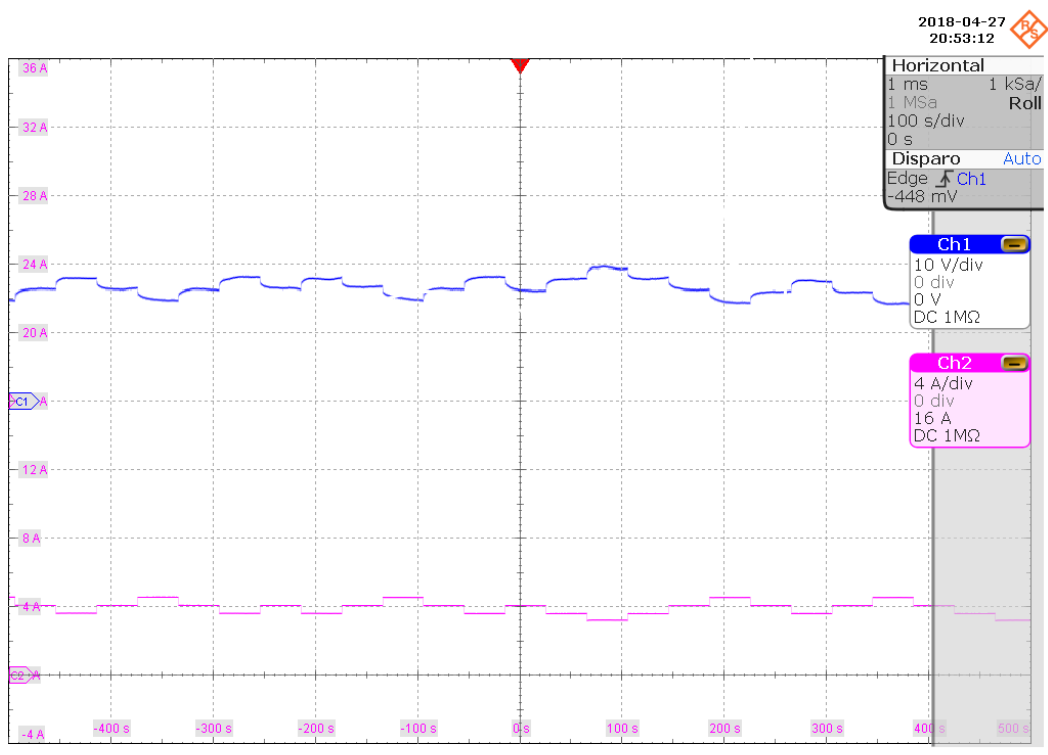

Figura 11. Prueba experimental de un MPPT con el emulador eólico

Fuente: elaboración propia

\section{CONCLUSIONES}

El presente documento presenta un banco para la simulación de turbinas eólicas, la implementación del convertidor DC/DC y un algoritmo MPPT, los cuales tienen el fin de probar el buen funcionamiento del emulador. El banco permite la emulación de las dinámicas de la captura de energía por medio del viento teniendo en cuenta el límite de Betz. Además, el banco permite emular cualquier turbina menor de $200 \mathrm{~W}$, con diferentes radios de pala y diferentes velocidades del viento, para así determinar según los datos meteorológicos de una zona, cuál es la mejor turbina para elegir, y realizar ajustes previos. Asimismo, el banco permite la prueba de nuevos algoritmos de extracción de máxima potencia que se implementen con nuevas técnicas, como por ejemplo IA, optimización, etc. También es posible pre-ajustar los algoritmos de control y optimización antes de concluir que son viables y proceder a su implementación en una aplicación real.

En trabajos futuros se recomienda realizar un sistema de extracción de energía utilizando la conversión de sistemas trifásicos en coordenadas D-Q y controlar un rectificador trifásico controlado; en este punto, el objetivo sería doble ya que no solo se realizaría el MPPT, sino también se aseguraría un factor de potencia unitario que permita mejorar aún más la eficiencia del sistema y probar múltiples algoritmos MPPT 
basados en Hill Climbing, técnicas de optimización avanzadas, redes neuronales y en el modelo matemático de la turbina.

También es de interés: desarrollar inversores para que el sistema pueda alimentar un conjunto de cargas AC en modo isla., desarrollar inversores que sean capaces de inyectar energía a una red o micro red $\mathrm{AC}$ y tener control de la potencia tanto activa como reactiva con el fin de utilizar el sistema eólico como una fuente de energía o como un dispositivo que mejore la calidad de la energía; es decir, como una fuente de transmisión flexible. Cuando se integran las energías renovables con bancos de baterías pueden ayudar al recorte de sobrepicos de demanda, al almacenar energía en momentos de bajo consumo energético y entregar energía en momentos de alto consumo energético. Tal concepto se conoce como "Peak Shaving" y permite aumentar las ganancias monetarias ya que la energía es más costosa en horas pico para los usuarios del sistema de tarificación de energía regulado.

\section{AGRADECIMIENTOS}

El presente artículo agradece al proyecto P14105 titulado Identificación, modelado y control de una turbina de viento por MPPT, desarrollado en la Línea de Investigación de Electrónica y Energías Renovables del Grupo de Investigación de Automática, Electrónica y Ciencias Computacionales del ITM, Colombia.

\section{REFERENCIAS}

[1] A. J. Chapman, B. C. Mclellan, and T. Tezuka, "Prioritizing mitigation e ff orts considering co-bene fi ts , equity and energy justice : Fossil fuel to renewable energy transition pathways," Appl. Energy, vol. 219, n. ${ }^{\circ}$ March, pp. 187-198, 2018.

[2] M. Narayana, K. M. Sunderland, G. Putrus, and M. F. Conlon, "Adaptive linear prediction for optimal control of wind turbines," Renew. Energy, vol. 113, pp. 895-906, 2017.

[3] J. Yan, Y. Feng, and J. Dong, "Study on dynamic characteristic of wind turbine emulator based on PMSM," Renew. Energy, vol. 97, pp. 731-736, 2016.

[4] O. Dahhani, A. El-jouni, and I. Boumhidi, "Assessment and control of wind turbine by support vector machines,” Sustain. Energy Technol. Assessments, vol. 27, pp. 167-179, 2018.

[5] A. Bonfiglio, F. Delfino, F. Gonzalez-longatt, and R. Procopio, "Steady-state assessments of PMSGs in wind generating units," Int. J. Electr. Power Energy Syst., vol. 90, pp. 87-93, 2017.

[6] A. Shafiei, B. M. Dehkordi, A. Kiyoumarsi, and S. Farhangi, "A Control Approach for Small - Scale PMSG - based WECS in the Whole Wind Speed Range," IEEE Trans. Power Electron., vol. 23, n. ${ }^{\circ}$ 12, pp. 9117-9130, 2017. 
[7] F. Martinez, L. C. Herrero, and S. De Pablo, “Open loop wind turbine emulator," Renew. Energy, vol. 63, pp. 212-221, 2014.

[8] J. C. Y. Hui, A. Bakhshai, and P. K. Jain, "An Energy Management Scheme with Power Limit Capability and an Adaptive Maximum Power Point Tracking for Small Standalone PMSG Wind Energy Systems," IEEE Trans. Power Electron., vol. 31, n. 7, pp. 4861-4875, 2016.

[9] J. F. Ruiz Murcia, J. Serna Cuenca, and H. J. Zapata Lesmes, Atlas de Viento de Colombia, Ideam-UPME., vol. 1, Colombia: Imprenta Nacional de Colombia, 2017.

[10] J. Castelló, J. M. Espí, and R. García-Gil, “Development details and performance assessment of a Wind Turbine Emulator,” Renew. Energy, vol. 86, pp. 848-857, 2016.

[11] F. Chejne, R. Smith, L. F. Rodr1, J. M. Mej1, and I. Dyner, "Simulation of wind energy output at Guajira , Colombia," Renew. Energy, vol. 31, pp. 383-399, 2006.

[12] E. G. Shehata, "A comparative study of current control schemes for a direct-driven PMSG wind energy generation system," Electr. Power Syst. Res., vol. 143, pp. 197-205, 2017.

[13] B. Yang et al., "Passivity-based sliding-mode control design for optimal power extraction of a PMSG based variable speed wind turbine," Renew. Energy, vol. 119, pp. 577-589, 2018.

[14] M. Rahimi, "Modeling, control and stability analysis of grid connected PMSG based wind turbine assisted with diode rectifier and boost converter," Int. J. Electr. Power Energy Syst., vol. 93, pp. 84-96, 2017.

[15] S. M. Muyeen, R. Takahashi, T. Murata, and J. Tamura, "Integration of an Energy Capacitor System With a Variable-Speed Wind Generator," IEEE Trans. Energy Convers., vol. 24, n. ${ }^{\circ}$, pp. 740-749, 2009.

[16] S. S. Dash and B. Nayak, "Control analysis and experimental verification of a practical dc-dc boost converter," J. Electr. Syst. Inf. Technol., vol. 2, n. 3, pp. 378-390, 2015.

[17] D. Zammit, C. S. Staines, A. Micallef, M. Apap, and J. Licari, "Incremental Current Based MPPT for a PMSG Micro Wind Turbine in a Grid-Connected DC Microgrid," in Energy Procedia, vol. 142, pp. 2284-2294, 2017.

[18] F. Alonge, M. Pucci, R. Rabbeni, and G. Vitale, "Dynamic modelling of a quadratic DC / DC single-switch boost converter," Electr. Power Syst. Res., vol. 152, pp. 130-139, 2017.

[19] J. P. Ram, N. Rajasekar, and M. Miyatake, "Design and overview of maximum power point tracking techniques in wind and solar photovoltaic systems: A review," Renew. Sustain. Energy Rev., vol. 73, pp. 1138-1159, 2017. 